\title{
High-throughput time group access MCR-SS-CSMA/CA for wireless ad hoc networks with layered-tree topology
}

\author{
Naoto Kobatake • Yasushi Yamao
}

Published online: 20 August 2011

(C) The Author(s) 2011. This article is published with open access at Springerlink.com

\begin{abstract}
Multi-hop packet transmission error due to packet collision is a serious issue for realizing large-scale wireless ad hoc networks. In order to solve the issues, a multicodereception spread-spectrum (MCR-SS)-CSMA/CA scheme is proposed as multiple access scheme for layered-tree networks. This scheme is further improved by adding a time group access (TGA) technique to gain higher interference reduction between nodes. The proposed TGA scheme divides the layered-tree network into upper subnets group and lower subnets group, thus reducing collision probability among subnets. Network throughput and data delivery ratio for the proposed scheme are analyzed by simulations based on the IEEE 802.15.4/ZigBee specification. Simulation results show that the proposed schemes can improve packet delivery ratio and network throughput remarkably.
\end{abstract}

Keywords Ad hoc network - Spread spectrum - CSMA . ZigBee

\section{Introduction}

Ubiquitous wireless networks are expected to be an important infrastructure in the future society. Most of the ubiquitous devices are assumed to be small and low-power, which

\footnotetext{
N. Kobatake

KDDI Corporation, 3-10-10 Iidabashi, Chiyoda-ku, Tokyo, 102-8460 Japan

e-mail: na-kobatake@kddi.com

Y. Yamao $(\bowtie)$

University of Electro-Communications, 1-5-1 Chofugaoka,

Chofu, Tokyo, 182-8585 Japan

e-mail: yamao@ieee.org
}

target data transmission applications such as tags and sensors. The functional goal of such networks is to provide a number of devices with ad hoc wireless connectivity and to self-organize a large-scale network with flexible topologies [1].

When constructing a large-scale wireless ad hoc network, multi-hop delay and packet error arise as serious issues. The delay increases as the number of hop counts increases. Packet error probability also increases as the number of hop counts increases [2]. Therefore the number of hops between any devices should be managed less than a permissible value. Also, autonomous data transmission using Carrier Sense Multiple Access with Collision Avoidance (CSMA/CA) often suffers from packet collision due to hidden terminals in the network.

A cluster-tree network [3] can reduce multi-hop delay by limiting hop counts by its layered structure. In the IEEE 802.15.4/ZigBee specification [4, 5], a network coordinator constructs and maintains the network [6]. However, the network frequently suffers from bottleneck at the network coordinator as a sink node and it limits whole data throughput in the network [7].

Several MAC schemes have been studied that introduced Spread Spectrum (SS) techniques to wireless ad hoc networks [8-10]. They suggest that the SS mitigates packet error due to hidden terminals and improves performance. However, many of their approaches are limited to protect important control message such as RTS/CTS [8] or beacon [9], and do not employ multicode reception (MCR) that utilizes full potential of SS Multiple Access (SSMA). Also, performance improvement resulting from a combination of SSMA and CSMA/CA has not been studied yet.

In this paper, SSMA employing multicode reception (MCR) and CSMA/CA are combined as new MAC protocols suitable for large-scale wireless ad hoc networks. 
The MCR scheme enables simultaneous reception of signals from multiple nodes in the same frequency radio channel with different orthogonal spreading codes. Thus, it is quite effective to mitigate bottleneck at the network coordinator and increase data throughput in the network.

However, in order to obtain the best performance by combining the MCR-SSMA and CSMA/CA, two protocol design issues should be solved. They are the spreading code assignment method and carrier sense method. First, a doublespreading code assignment method for cluster-tree networks is proposed to enable MCR in each subnet as well as to reduce interference among subnets. Next, a carrier sense method after despreading is proposed in order to improve independency of MCR in each subnet [11].

The proposed MCR-SS-CSMA/CA protocol still suffers from interference among subnets. Therefore, the protocol is further improved by adding a time group access (TGA) technique to gain higher interference reduction between subnets. The proposed scheme divides a cluster-tree network into upper subnets group and lower subnets group and assign two groups with different time slots. Thus, collision probability among subnets is reduced, which improves packet transmission success ratio and increases whole network throughput. Hereafter it is referred to as TGA-MCR-SS-CSMA/CA.

The improvements by the proposed schemes on the throughput and packet delivery ratio are evaluated by simulations. The remainder of the paper is structured as follows; the proposed protocols are described in Sect. 2. The simulation setup and evaluation results for a 37 nodes cluster-tree network are shown and discussed in Sect. 3. The paper ends with conclusions and outlook in Sect. 4.

\section{MCR-SS-CSMA/CA}

The cluster-tree network shown in Fig. 1 is organized by connecting subnets. A Network Coordinator (NC) builds and manages the network to form a layered structure. A subnet with star topology consists of a parent node, either a $\mathrm{NC}$ or a router, and child nodes. Child nodes are either end devices or routers that connect with lower layer subnets. A router node communicates with both an upper layer node and its child nodes. The network topology is suitable for realizing large-scale wireless ad hoc networks with scalability while controlling multi-hop counts.

Since child nodes can synchronize to a parent router node with a beacon transmitted by the parent router node, each subnet operates as a synchronous network. Thus, intermittent operation is possible in the cluster tree network and low power consumption is achieved [7].

\subsection{SS-CSMA/CA and spreading code assignment}

The multiple access method proposed in the paper is a combination of multicode SSMA and CSMA/CA. Although

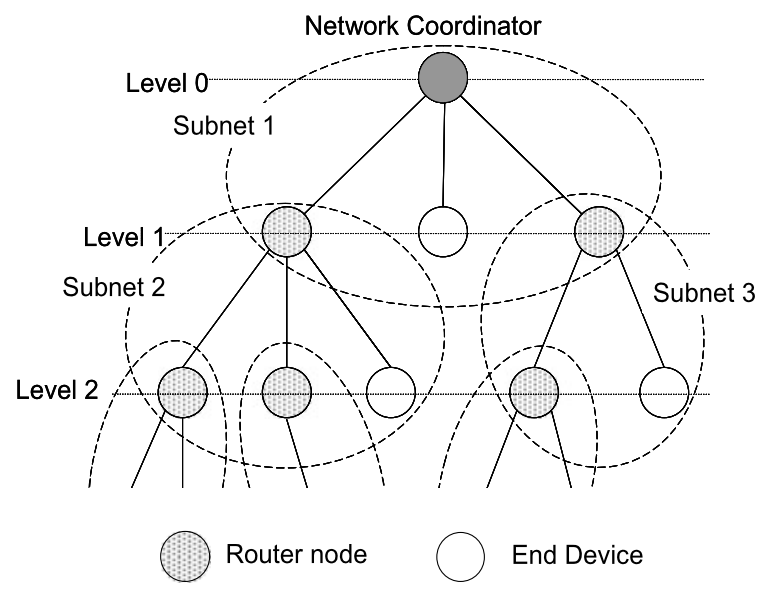

Fig. 1 Cluster-tree network topology

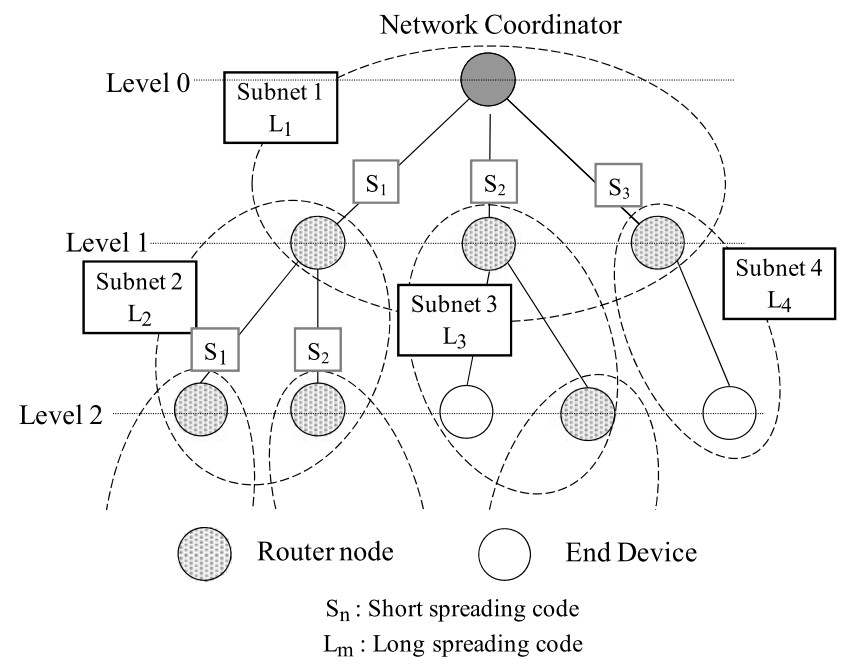

Fig. 2 Spreading code assignment for cluster-tree network

SSMA can mitigate packet error due to hidden terminals and improve performances, improvement gained by SS depends on spreading code assignment method.

The spreading code assignment method proposed for cluster-tree networks is shown in Fig. 2. A double-spreading code is employed which is obtained by multiplying short and long spreading codes. The diagram of a double-spreading code for a spreading factor (SF) of 8 as an example is shown in Fig. 3. Long spreading codes are assigned subnet by subnet, and short codes are assigned to nodes in a subnet. When the cluster-tree network is constructed, parent node assigns the spreading codes to its child nodes. Then each node generates its double-spreading code by multiplying its short code and the long code of the subnet.

Since nodes in a subnet are synchronized and assigned with the same long spreading code, orthogonal codes can be used as the short codes. It enables simultaneous transmission from multiple nodes in the subnet using the same frequency radio channel. For the low-power ubiquitous ad hoc 
Fig. 3 Double-spreading code $(\mathrm{SF}=8)$

Fig. 4 Multicode reception for SS-CSMA/CA
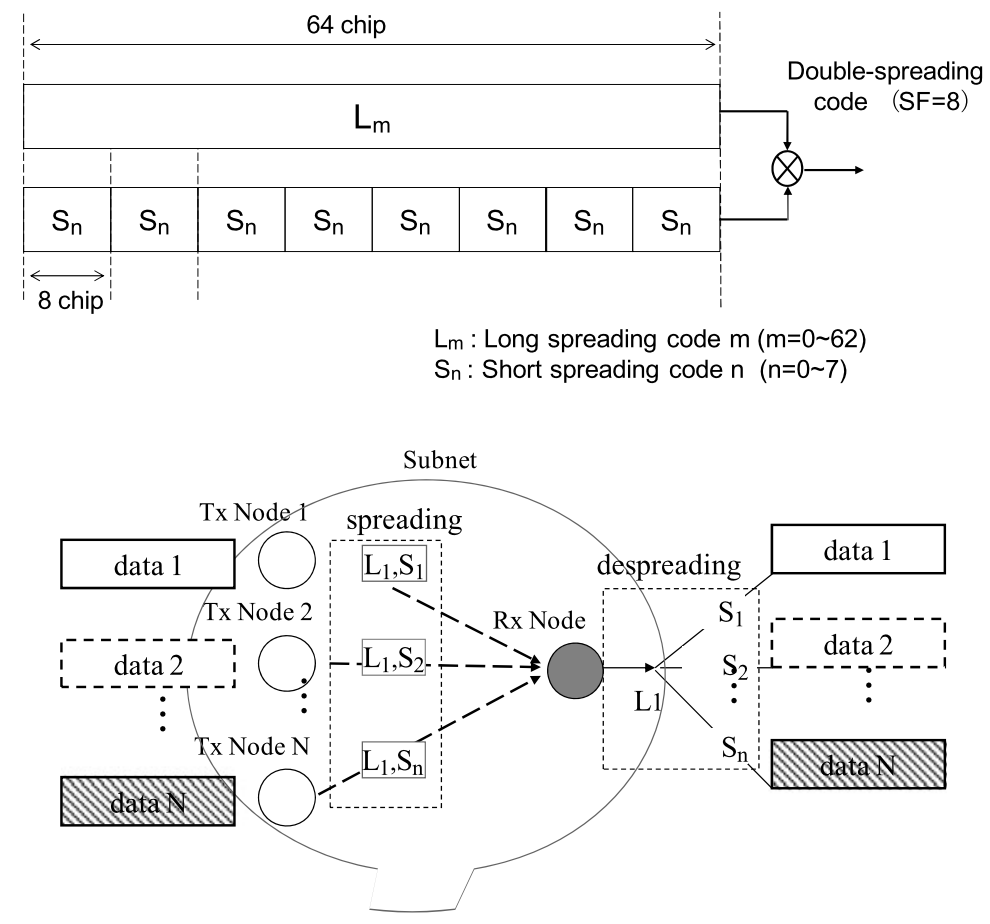

networks, communication range for each node is short compared to the distance determined by chip duration. In this case, degradation of orthogonality due to different propagation delay can be ignored in the subnet.

For nodes belonging to different subnets, transmitted signals are not orthogonalized. Nevertheless, interference power from other terminals is reduced by $1 / \mathrm{SF}$ after despreading because different long spreading codes are used. However, considering received power fluctuation due to fading in wireless environments, packet collision due to simultaneous transmission still happen. In order to avoid it, CSMA/CA is combined in the proposed method.

\subsection{Multicode reception (MCR)}

Multicode reception (MCR) is employed to mitigate bottleneck at the NC and enhance data throughput in the network. Multicode reception for the proposed SS-CSMA/CA is shown in Fig. 4. A receiver node performs despreading for all spreading codes that may be used in the subnet, and tries demodulation of the received signals. Thus, simultaneous reception of the multiplexed signals with different orthogonal spreading codes is achieved. The maximum number of multiplexed signals $N$ is equal to the number of available orthogonal codes, which is determined by SF.

\subsection{Carrier sense method}

When the CSMA/CA is combined with SS, the following two carrier sense (CS) methods will be the candidates.
(1) CS by normally detected power (normal CS).

(2) CS by detected power after despreading (CS after despreading).

With the first method, a transmitting node will stop its transmission when it detects other transmitter's signals from the same subnet with different orthogonal codes. This makes the node losing chance of transmission, spoils the advantage of MCR and decreases data throughput due to unnecessary waiting.

When performing CS after despreading with an assigned spreading code, a transmitting node judges the existence of other transmitter's signals using the same spreading code. If the number of child nodes in a subnet is more than SF, the same spreading code is assigned to child nodes. In this case, simultaneous transmission by the nodes using the same spreading code can be avoided by the CS after despreading.

By combining the CS after despreading and MCR, the proposed MCR-SS-CSMA/CA enables multiple access in a subnet and permits multiple communications at different subnets. Consequently, probability of transmission waiting is reduced and the data throughput in the network will be improved.

\subsection{Time group access (TGA) SS-CSMA/CA}

Packet collision among subnets significantly affects packet reception success probability in the cluster-tree networks. This situation can be improved by reducing the number of subnets that interferes each other at the same time. Therefore 
a time group access (TGA) scheme is proposed to combine with the MCR-SS-CSMA/CA.

The idea of time group access in a cluster-tree network is shown in Fig. 5. In the figure, the network coordinator (NC) is the parent node of subnet 1 . Three router nodes are the child nodes in the subnet 1 . At the same time, they are parent nodes of different subnets, subnet 2, 3 and 4. If data packets are generated at end devices and their destination is the NC, they are forwarded by its parent routers. Since the end devices and router nodes in different subnets 2, 3 and 4 transmit data packet independently, packet collision occurs among them when they are in the condition of "temporary hidden terminal" due to fading.

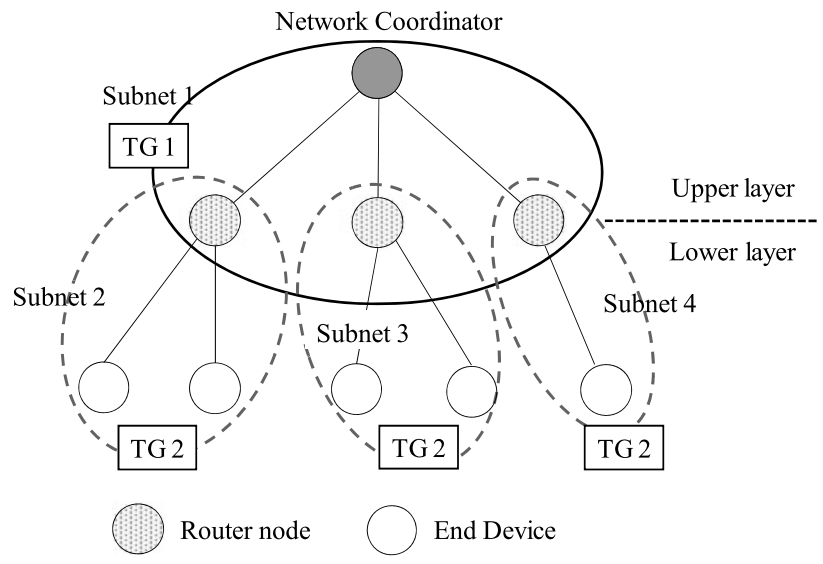

(a) Two TGA grouping.

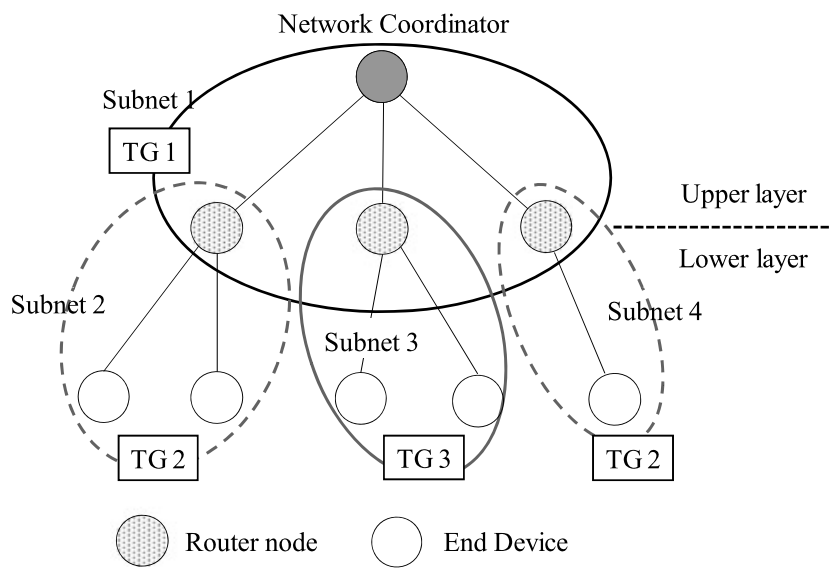

(b) Three TGA grouping

Fig. 5 Time group access in cluster-tree network
In order to reduce the collision probability, TGA is introduced in the following two steps;

Step (1): Two-group TGA

Separate upper subnet 1 from lower subnets $(2,3,4)$ by setting TG1 for subnet 1 and TG2 for subnets 2, 3, 4 .

Step (2): Three-group TGA

In addition to the step (1), divide lower subnets 2, 3 and 4 into two groups and set TG1 for subnet 1, TG2 for some lower subnets, and TG3 for other lower subnets.

By the step (1), collisions between routers and end nodes can be avoided. By the step (2), collisions between end nodes of different subnet groups will be decreased in addition to the effect of the step (1). Figure 5(a) shows the step (1) TGA and Fig. 5(b) shows an example of the step (2) TGA.

Figure 6 shows a communication period assignment example for the step (2) TGA. Time periods of $t_{1}, t_{2}$ and $t_{3}$ are assigned to TG1, TG2 and TG3, respectively. They do not overlap each other. In case of step (1) TGA, TG2 and TG3 are merged into TG2. The time period for the merged TG2 is also represented by $t_{2}$. Nodes in Each TG perform communication during the assigned period. Since most of ubiquitous devices are required to be low-cost and low-power, node devices can have only one transceiver. This means that routers cannot communicate with the upper and lower layers at the same time. The TGA grouping scheme satisfies this condition.

TG grouping or communication period assignment for each subnet is determined by the NC when the cluster tree network is constructed. Time alignment for each subnet is achieved referring the beacon signal from the parent node. In the layered-tree structure, a router node synchronizes to the beacon of its upper layer router or the NC. Thus, whole network synchronizes to the frame timing of the NC.

Interference among subnets can be reduced by decreasing the number of subnets within a TG by introducing more TGs. However, there is trade-off between improvement in interference reduction and division loss in time domain access. If a constant superframe duration that includes all TG communication periods is given, the division loss is caused by two reasons. One is regarding to ratio of data packet size and communication period. If a data packet is far shorter than the communication period, the division loss of this kind can be neglected. The other is unbalance of communication period lengths among TGs regarding the traffic unbalance
Fig. 6 Communication period assignment example

\begin{tabular}{|c|c|c|c|}
\hline & \begin{tabular}{|c|}
$\begin{array}{c}\text { Communication } \\
\text { Period } 1\end{array}$ \\
\end{tabular} & \begin{tabular}{|c|}
$\begin{array}{c}\text { Communication } \\
\text { Period 2 }\end{array}$ \\
\end{tabular} & \begin{tabular}{|c|}
$\begin{array}{c}\text { Communication } \\
\text { Period } 3\end{array}$ \\
\end{tabular} \\
\hline Upper layer communication & TG1 & & \\
\hline Lower layer communication & & TG2 & TG3 \\
\hline
\end{tabular}


Table 1 Radio transmission and propagation parameters

\begin{tabular}{ll}
\hline RF band & $2.4 \mathrm{GHz}$ \\
Data rate & $250 \mathrm{kbit} / \mathrm{s}$ \\
SF & 8 \\
Tx power & $0 \mathrm{dBm}$ \\
Noise level & $-110 \mathrm{dBm}$ \\
Receiver sensitivity & $-94 \mathrm{dBm}$ \\
Required SINR for reception & $10 \mathrm{~dB}$ \\
Propagation loss exponent & 2.5 \\
Fading & Rayleigh \\
Data packet size & 70 byte \\
\hline
\end{tabular}

for TGs. The amount of traffic for TGs should be balanced to obtain the best performance, which can be achieved by adjusting $t_{1}, t_{2}$ and $t_{3}$. The NC can monitor the traffic from subnets and assign the best communication periods among TGs.

\section{Performance evaluation}

Performance of the proposed schemes in the cluster-tree network was evaluated by computer simulation using an original C program. The IEEE 802.15.4/ZigBee specification was referred to create a cluster-tree network in the simulation. Physical layer and the slotted CSMA/CA medium access control (MAC) layer were based on the IEEE 802.15.4 [12].

The IEEE 802.15.4 physical layer employs a DS-SS with $\mathrm{SF}$ of eight for mitigating interference. However, since the modulation encoding is common for all nodes, interference mitigation effect with the DS-SS cannot be expected when the cluster tree network performs synchronous operation. In the simulation, the performance of the proposed method was examined by substituting the specified modulation codes to the double-spreading codes with the same SF. The Walsh codes with a code length of 8 are employed as the short spreading code set, and the pseudorandom sequences with a code length of 64 are employed as the long spreading code.

Radio transmission parameters are listed in Table 1. RF band, data rate, and TX power are referred to the IEEE 802.15.4 physical layer specification. Noise level, receiver sensitivity and required SINR for reception are typical values for the IEEE 802.15.4 chip set. A communication period is based on the length of the superframe of IEEE 802.15.4, which is $15.36 \mathrm{~ms}$ (480 byte).

\subsection{Basic performance in a subnet}

In order to evaluate the fundamental performance of proposed method, a simulation for star subnet was performed. Fifty transmitting nodes were randomly placed in a circle area with a receiving node at the center. Each transmitting

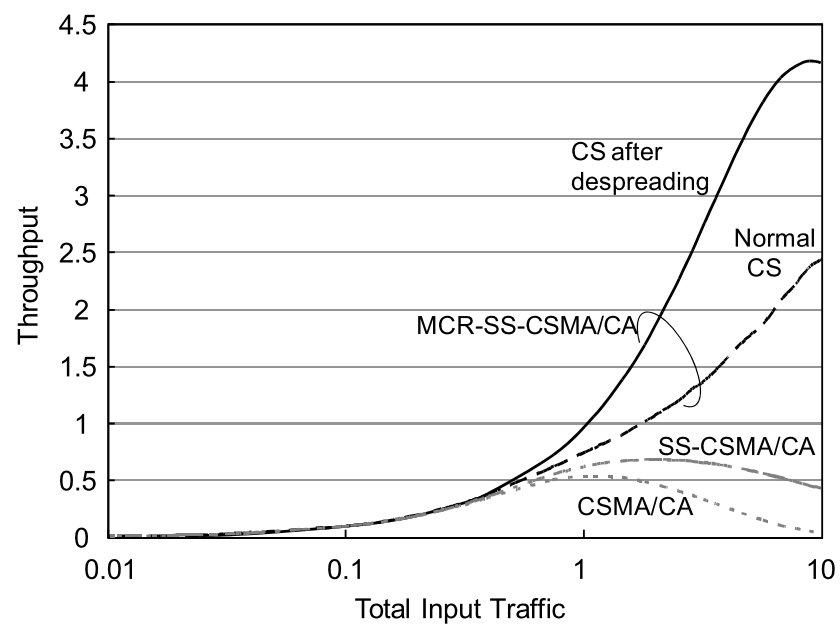

Fig. 7 Throughputs of MAs for a star subnet

node was randomly assigned one of eight Walsh short codes and a common long code. Since the number of nodes is more than SF, a short code was assigned to multiple nodes.

Each node generated data packets with uniform traffic according to a Poisson distribution and sent them to the receiving node by single-hop. The normalized network throughput is defined as the total time occupancy ratio of successfully received data packets at the receiving node. The CS level was basically set at $-92 \mathrm{dBm}$, since the CS level is specified by either the same as or more than receiver sensitivity.

Figure 7 shows the throughput performance for the proposed MCR-SS-CSMA/CA, single-code reception SSCSMA/CA and CSMA/CA. Two CS methods for the proposed MCR-SS-CSMA/CA are compared in the figure. CS level and area radius were set at $-92 \mathrm{dBm}$ and $10 \mathrm{~m}$, respectively. The horizontal axis shows total traffic from 36 nodes. Comparing throughputs for CSMA/CA and single-code reception SS-CSMA/CA, there is no significant improvement by SS. However, throughputs for the proposed MCR-SSCSMA/CAs are remarkably improved. These results mean that not only SS but MCR contributes much to increase throughput. The best result was obtained by the proposed MCR-SS-CSMA/CA with CS after despreading. The peak throughput of the proposed method is 8 times of CSMA/CA.

Figure 8 compares the total throughputs with two CS levels for the proposed MCR-SS-CSMA/CA with CS after despreading. The throughputs are better with the lower CS level of $-92 \mathrm{dBm}$ for both area radius of 10 and $30 \mathrm{~m}$. These results show that higher CS sensitivity prevents collision of packets with the same short spreading code. Packet collision happens when the distance of two transmitting nodes exceeds a CS range. Larger the radius of the subnet, higher the number of node pairs in out of CS range. Then probability of collisions increases with the subnet radius.

Figure 9 shows the peak throughput versus area radius for the proposed and conventional MAs with different CS 


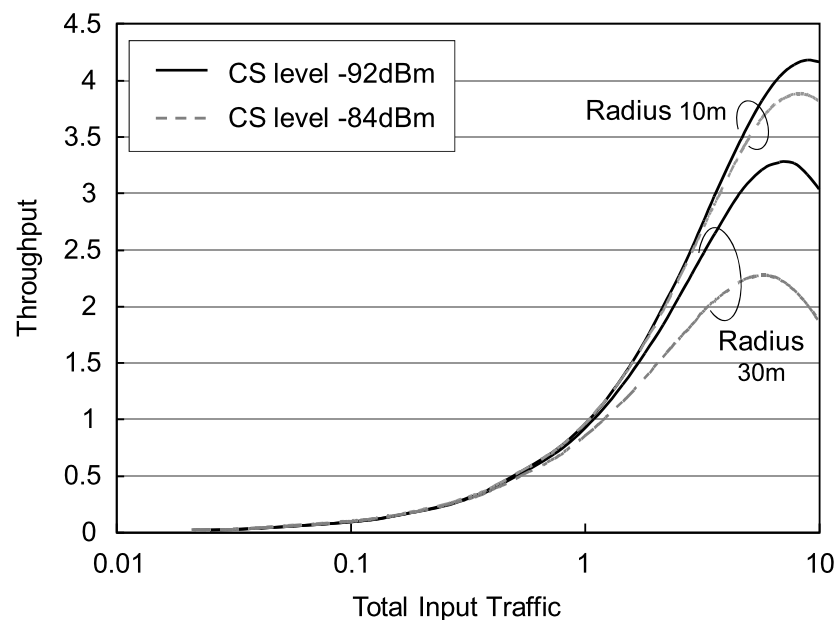

Fig. 8 CS level vs. throughput for MCR-SS-CSMA/CA with CS after despreading

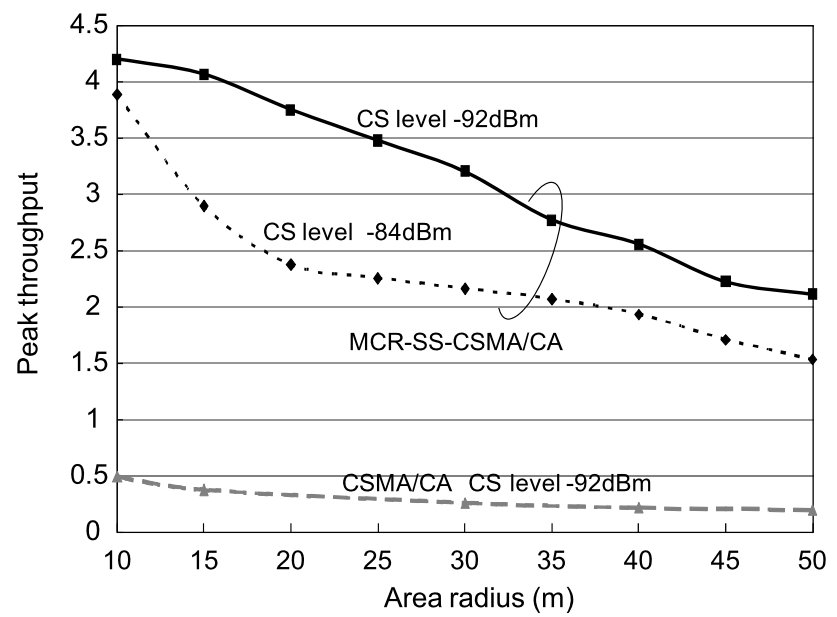

Fig. 9 Peak throughput versus area radius

methods and CS levels. The result shows that the peak throughput gradually decreases for all MAs, however, the proposed MCR-SS-CSMA/CA with CS after despreading and $\mathrm{CS}$ level of $-92 \mathrm{dBm}$ keeps the highest throughput. Hereafter, the CS level of $-92 \mathrm{dBm}$ is used for evaluation.

\subsection{Cluster-tree network performance}

A cluster-tree network model shown in Fig. 10 was used for network level evaluation. 37 nodes were geographically located on a regular triangle lattice with an adjacent node distance of $10 \mathrm{~m} .36$ nodes other than a NC were arranged around the NC, and the number of router nodes is eight. The $\mathrm{NC}$ and eight routers form the central subnet. Routers are the parent nodes of the surrounding eight subnets and each router has three or four child nodes (end devices). Since the number of child nodes in the subnets is less than SF, assignment of the spreading codes is not duplicated.

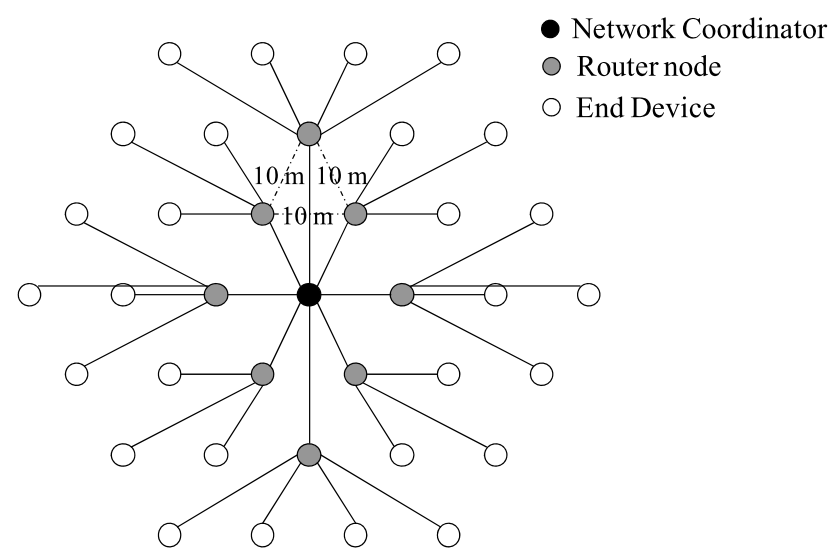

Fig. 10 Cluster-tree network model with 37 nodes



Fig. 11 Two-group TGA-SS-CSMA/CA

Each end node generates uniform data packet traffic according to a Poisson distribution. All data packets are addressed to the NC and relayed by routers. Each router is equipped with a transmitting queue buffer with the size of 200 packets.

The performances of 2-group and 3-group TGA-MCRSS-CSMA/CA are evaluated as well as MCR-SSCSMA/CA without TGA. Figure 11 shows the grouping of subnets for 2-group TGA-SS-CSMA/CA. The 2-group TGA-SSCSMA/CA divides the cluster-tree network into upper layered subnet group (TG1) and lower layered subnets group (TG2). The length of each communication period assigned to TG is equal $\left(t_{1}=t_{2}=15.36 \mathrm{~ms}\right)$.

In the 3-group TGA, the cluster-tree network is divided into upper layered subnet group (TG1) and lower layered subnet groups (TG2 and TG3). When TG2 and TG3 are assigned to the lower layered subnets, each neighboring subnet is assigned a different TG as shown in Fig. 12. The length of communication periods assigned to TGs are equal $\left(t_{1}=t_{2}=t_{3}=15.36 \mathrm{~ms}\right)$.

Evaluation criteria are total network throughput and data delivery ratio. The total network throughput is defined as 


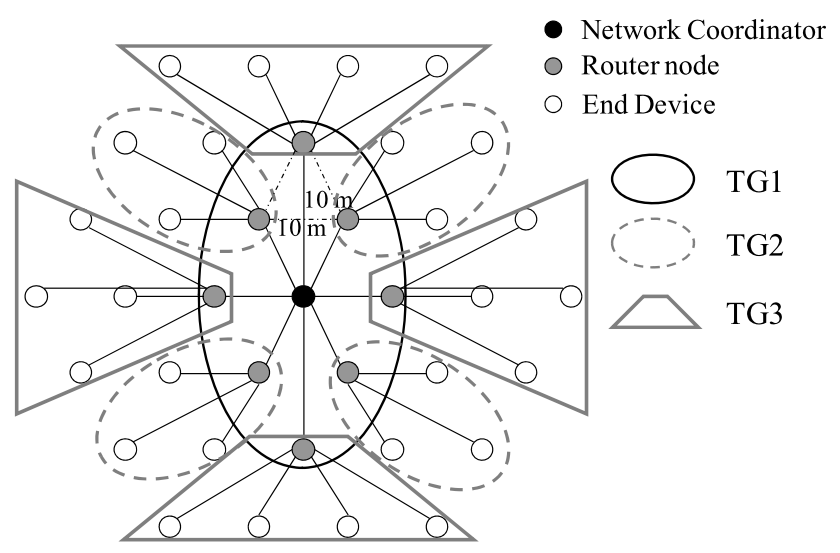

Fig. 12 Three-group TGA-SS-CSMA/CA

the sum of time occupancy ratio of data packets that are successfully received at the NC. In the multicode reception system, router nodes can send data packets in parallel with different spreading codes and the network coordinator can receive them independent each other. This is equivalent to use multiple transmission channels. Thus, the whole network throughput as the sum of channel capacity can exceed one. The data delivery ratio is defined as the ratio in number of successfully received packets at the NC to generated packets.

Figure 13 compares the network throughput for four schemes, MCR-SS-CSMA/CA with 3-group TGA, 2-group TGA, without TGA (MCR-SS-CSMA/CA), and CSMA/CA. The horizontal axis shows the total input traffic generated at all end nodes. The proposed TGA-SS-CSMA/CA schemes remarkably improve the throughput performance when the input traffic is higher than 0.2. The 3-group TGA-SS-CSMA/CA gains the highest throughput among these schemes. Peak throughput for the proposed TGASS-CSMA/CA schemes is around twice of the MCR-SSCSMA/CA without TGA.

Figure 14 shows the packet delivery ratios for the four schemes. Packet delivery ratio is also improved by the proposed TGA-SS-CSMA/CA schemes for all input traffic. The 3-group TGA-SS-CSMA/CA provides the highest packet delivery ratios among these schemes. These results show that packet collision loss is kept low with the TGA-SSCSMA/CA schemes.

Figure 15 shows the packet delivery ratio from routers to the NC. The TGA-SS-CSMA/CAs have very good packet delivery ratio close to one. On the other hand, MCR-SSCSMA/CA without TGA suffers from packet loss for the input traffic larger than 0.3. From the analysis of simulation results, there are two reasons for the packet loss for MCRSS-CSMA/CA without TGA. The first one is the loss due to interference from end devices. There is no interference from other router nodes because orthogonal spreading codes are employed in the upper layer subnet 1 . Contribution of this

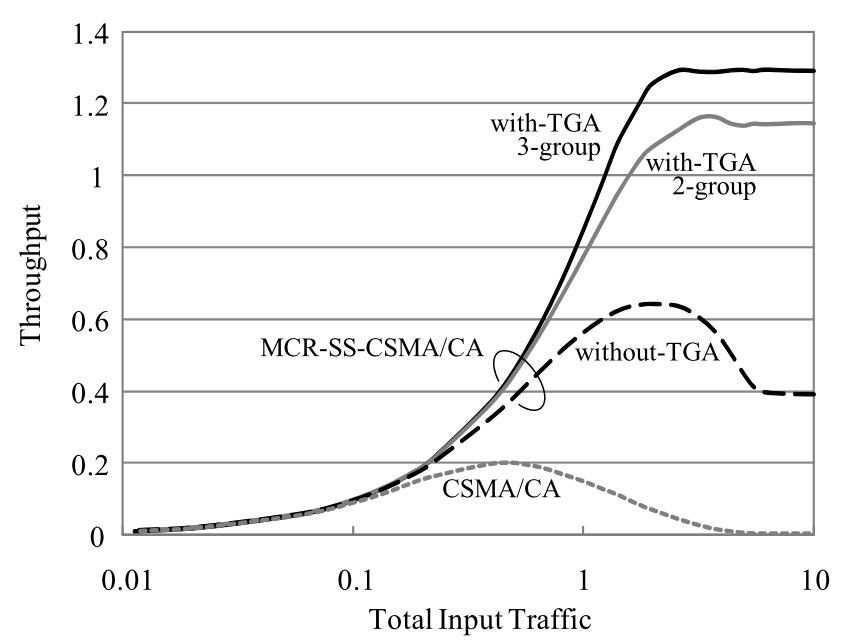

Fig. 13 Throughput for TGA-SS-CSMA/CA schemes

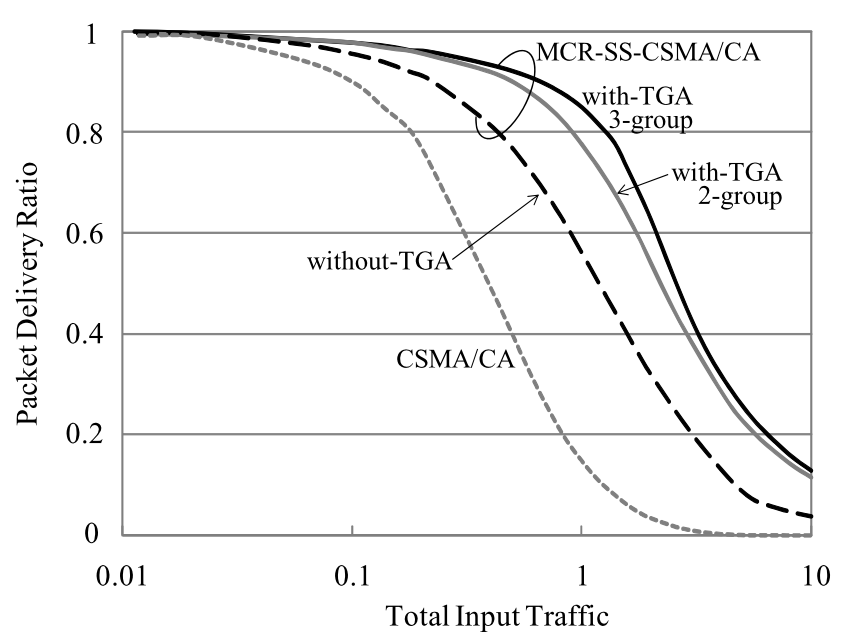

Fig. 14 Packet delivery ratio for TGA-SS-CSMA/CA schemes

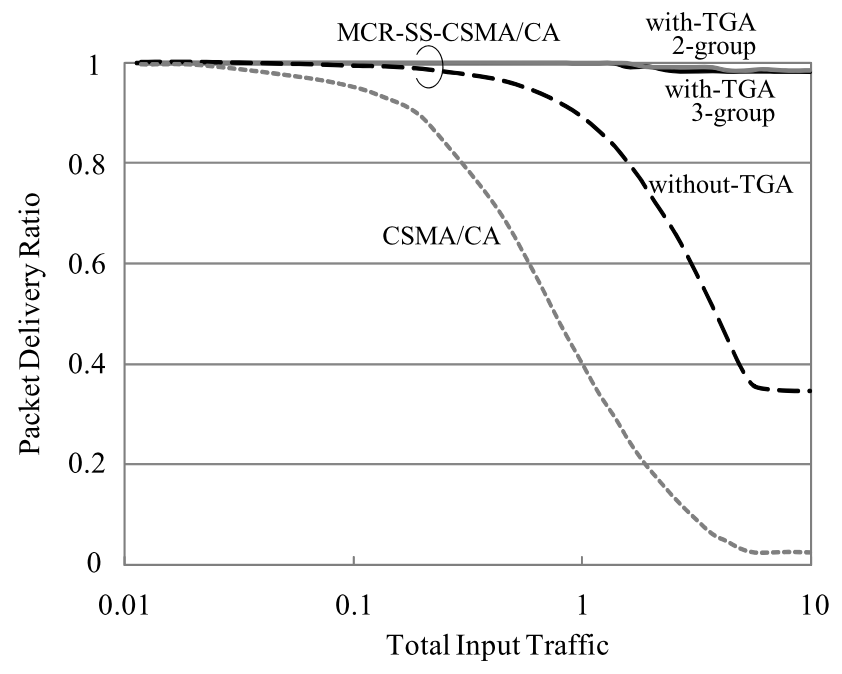

Fig. 15 Packet delivery ratio from routers to NW coordinator 
loss is not great because distances from end devices to the $\mathrm{NC}$ are larger than that from router nodes to the NC.

The other important reason of the packet loss is buffer overflow at the router nodes due to transmission waiting according to the CSMA/CA mechanism. In the simulation, each router is equipped with a transmitting queue buffer with the size of 200 packets. However, the routers and end devices have the same priority for the transmission, whereas the number of the end devices is greater than that of router nodes. Therefore, probability to get priority for transmission is small for the router nodes without TGA. Consequently, buffer overflow happens at the routers. With the proposed TGA-SS-CSMA/CA, the communication period for the router nodes is separated from that for the end devices. Thus, independent transmission of router nodes is guaranteed, which enables to remove the throughput bottleneck reason from router nodes.

Figure 16 shows the Packet delivery ratio from end devices to router nodes. Packet delivery ratios for the proposed TGA-SS-CSMA/CA schemes are higher than MCR-

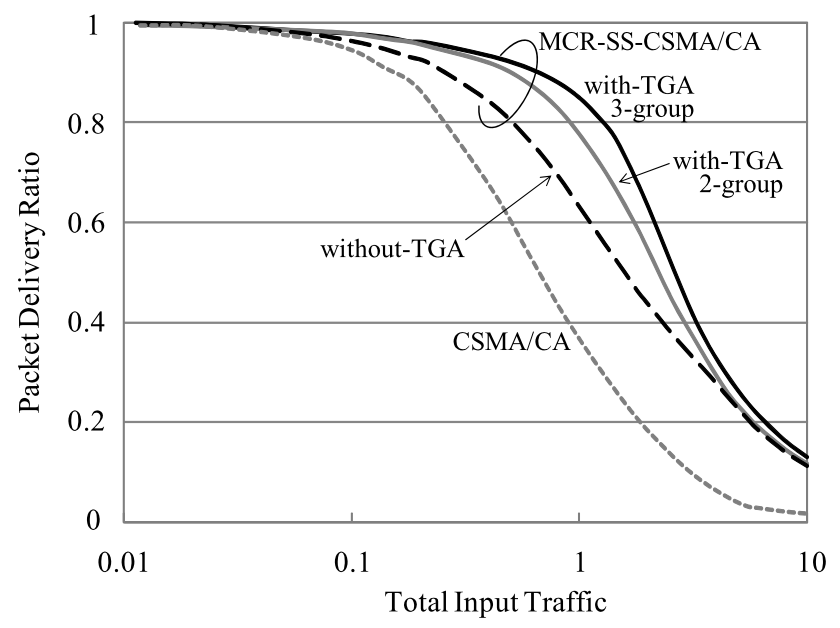

Fig. 16 Packet delivery ratio from end devices to routers
SS-CSMA/CA without TGA. Also, the proposed 3-group TGA-SS-CSMA/CA gains the highest packet delivery ratio among these methods.

Performance improvement with the 3-group TGA-SSCSMA/CA depends on how lower layer subnets are grouped into TG2 and TG3. Figure 17 shows dependence of the throughput on the lower layer subnet groupings. The best throughput can be obtained by the grouping shown in Fig. 12. If lower layer subnets are assigned random to TG2 and TG3, the peak throughput is similar to that for 2-group TGA-SS-CSMA/CA.

\section{Conclusion}

MCR-SS-CSMA/CA has been proposed as a new MAC protocol suitable for large-scale wireless ad hoc networks with layered structure. In order to mitigate bottleneck and increase data throughput, multicode reception is applied to the network coordinator. A double-spreading code assignment method and a carrier sense method after despreading has been proposed to obtain full potential of the multicode reception in each subnet, as well as to reduce interference among subnets.

In order to further mitigate interference among subnets, the MCR-SS-CSMA/CA has been improved by adding a time group access (TGA) technique. Throughput and data delivery ratio for the proposed schemes are analysed by simulations for a 37-nodes cluster-tree network and based on the IEEE 802.15.4/ZigBee specification. Simulation results show that the throughput and the data delivery ratio are remarkably improved by the proposed schemes.

It is concluded that proposed MCR-SS-CSMA/CA schemes are effective for mitigating packet loss and improving network performance in large-scale wireless ad hoc networks.
Fig. 17 Throughput for 3-group TGA-SS-CSMA/CA with different groupings

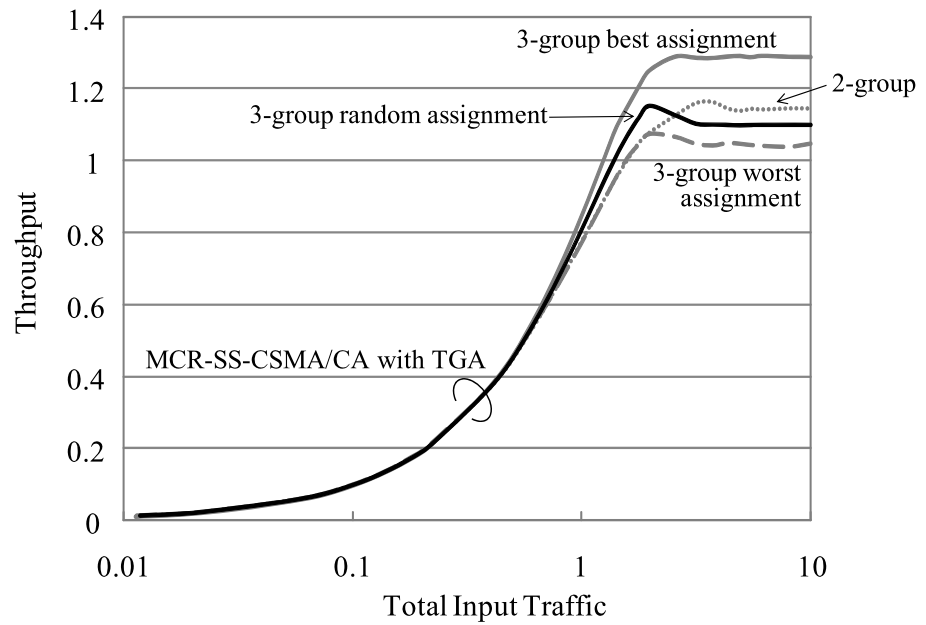


Open Access This article is distributed under the terms of the Creative Commons Attribution Noncommercial License which permits any noncommercial use, distribution, and reproduction in any medium, provided the original author(s) and source are credited.

\section{References}

1. Zheng, J., \& Lee, M. J. (2004). Will IEEE 802.15.4 make ubiquitous networking a reality? A discussion on a potential low power, low bit rate standard. IEEE Communications Magazine, 42(6), 140-146.

2. Nagao, K., Kadowaki, Y., \& Yamao, Y. (2009). Multi-hop transmission performance of cognitive temporary bypassing for wireless ad hoc networks. In Proc. IEEE CCNC2009, Las Vegas, Jan. 2009.

3. Hester, L., Huang, Y., Allen, A., Andric, O., \& Chen, P. (2002). neuRFon netform: a self-organizing wireless sensor network. In Proc. 11th IEEE int'l conf. computer comm. and networks conf., Oct. 2002.

4. ZigBee Alliance, http://www.zigbee.org.

5. ZigBee Network Specification, V1.0, Dec. 2004.

6. Zheng, J., Lee, M. J., \& Anshel, M. (2006). Toward secure low rate wireless personal area networks. IEEE Transactions on Mobile Computing, 5(10), 1361-1373.

7. Yamao, Y., \& Takagishi, S. (2008). Time shift grouping access in IEEE 802.15.4 MAC beacon mode for layered-tree networks. In Proc. IEEE CCNC2008, Las Vegas, Jan. 2008.

8. Joa-Ng, M., \& Lu, I-T. (1999). Spread spectrum medium access protocol with collision avoidance in mobile ad-hoc wireless network. In Proc. IEEE infocom 1999 (Vol. 2, pp. 776-783).

9. Yeh, C.-H. (2004). Spread spectrum techniques for solving MAClayer interference issues in mobile ad hoc networks. In Proc. IEEE VTC 2004-Spring, Milan (pp. 1339-1344).

10. Andrews, J. G., Weber, S. \& Haenggi, M. (2007). Ad hoc networks: to spread or not to spread? IEEE Communications Magazine, 14, 84-91.

11. Kobatake, N., \& Yamao, Y. (2009). Multi-code SS-CSMA/CA for cluster-tree wireless ad hoc networks. In IEEE ISWCS2009, Sept. 2009.

12. IEEE 802.15.4 Standard (2000). Wireless medium access control (MAC) and physical layer (PHY) specifications for low-rate wireless personal area networks (LR-WPANs), Oct. 2000.

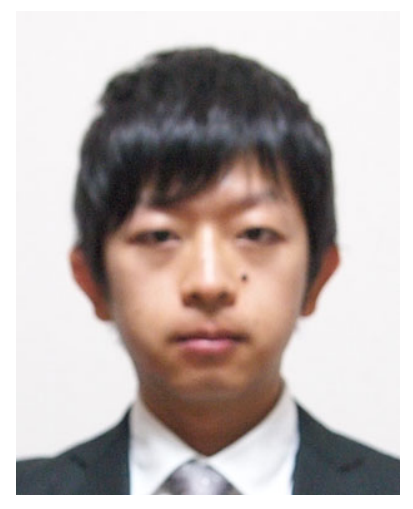

Naoto Kobatake received his B.S. and M.S. degrees in Electrical Communication Engineering from the University of Electro-Communications in 2008 and 2010, respectively. $\mathrm{He}$ is now with KDDI Corporation.

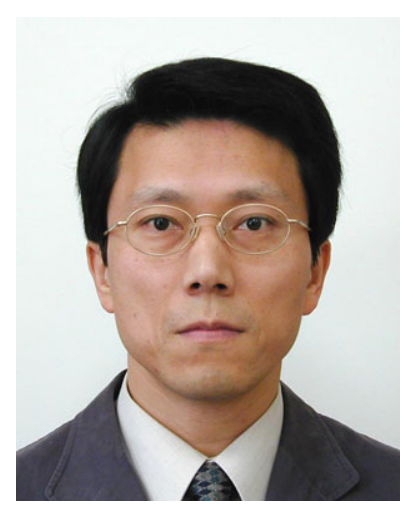

Yasushi Yamao received his B.S., M.S., and Ph.D. degrees in electronics engineering from Kyoto University, Kyoto, Japan, in 1977, 1979, and 1998, respectively. In 1979, he joined the Nippon Telegraph and Telephone Corporation (NTT) Laboratories, Japan, where his major activities included research on the GMSK modulator/demodulator and GaAs RF ICs for digital mobile communications, and development of PDC digital cellular handheld phones. In 1993, he moved to NTT DoCoMo, Inc. In 2005, he moved to the University of Electro-Communications as a professor of the Advanced Wireless Communication Research Center (AWCC). His current interests focus on wireless ubiquitous communication networks and protocols, as well as high-efficiency wireless circuit technologies. $\mathrm{He}$ is Fellow of IEICE and member of IEEE. He served as Vice President of the IEICE Communications Society (2003-2004), Chairman of the IEICE Technical Group on Radio Communication Systems (20062008) and Chief Editor of the IEICE Communication Society Magazine (2008-2010). He is now the Vice Chairman of the IEEE VTS Japan Chapter. 